\title{
DISTRIBUCIÓN ESPACIAL, COMPOSICIÓN Y ESTRUCTURA DE UN MATORRAL EN EL NORESTE DE MÉXICO
}

\section{SPATIAL DISTRIBUTION, COMPOSITION AND STRUCTURE OF A THORNSCRUB IN NORTHEASTERN MÉXICO}

\section{Carlos A. Mora-Donjuán, Enrique Buendía-Rodríguez², Ernesto A. Rubio-Camacho, Eduardo Alanís-Rodríguez ${ }^{1}$ y Eduardo J. Treviño-Garza'}

\begin{abstract}
'Facultad de Ciencias Forestales, Universidad Autónoma de Nuevo León. Km 145 Carretera Linares-Cd. Victoria. 67700, Apartado Postal 41,Linares, N.L., México. ${ }^{2}$ Campo Experimental Valle de México, Instituto Nacional de Investigaciones Forestales, Agrícolas y Pecuarias (INIFAP). Km. 13.5 Carretera los Reyes-Texcoco. 56250, Apartado Postal 10. Coatlinchán, Texcoco, Estado de México, México. ${ }^{3} \mathrm{C}$. E. Centro Altos de Jalisco, INIFAPCIRPAC. Km. 8 Carretera Tepatitlán-Lagos de Moreno. 47600, Apartado Postal 56. Tepatitlán de Morelos, Jalisco.
\end{abstract}

*Autor de correspondencia (biologomora@gmail.com)

\section{RESUMEN}

En este estudio se describe la distribución espacial, composición y estructura arbórea y arbustiva de un área del matorral espinoso tamaulipeco (MET), mediante los índices de agregación $(R)$, uniformidad ángulos $(W i)$, segregación (S), mezcla de especies (Mi), diferenciación dimensional (Ti) y dominancia dimensional $(U i)$, para que sirvan de base para establecer programas de restauración ecológica. Se ubicó un área del MET sin remoción de la vegetación y sin registro de actividad productiva en 28 años. Se establecieron cuatro sitios de $40 \times 40 \mathrm{~m}$ Para cada árbol y arbusto $\left(d_{0.10} \geq 5\right.$ $\mathrm{cm}$ ) se determinó la especie y midió el diámetro de copa, altura total, distancia y azimut con respecto al centro del sitio. Los resultados de los índices fueron: para agregación $R_{1}=0.76, R_{2}=0.43, R_{3}=0.50$ y $R_{4}=0.83$; uniformidad de ángulos $W i_{1}=0.57 \pm 0.20, W i_{2}=0.61 \pm 0.24, W i_{3}=0.59 \pm 0.23$ y Wi $=0.52$ \pm 0.20 ; segregación $S_{1}=0.24, S_{2}=0.68, S_{3}=0.66$ y $S_{4}=0.04$, mezcla de especies $\mathrm{Mi}_{1}=0.73 \pm 0.24, \mathrm{Mi}_{2}=0.47 \pm 0.35, \mathrm{Mi}_{3}=0.52 \pm 0.34 \mathrm{y} \mathrm{Mi}_{4}=0.84 \pm 0$; diferenciación diamétrica $T_{13.4}=0.49 \pm 0.35$ y $T_{2}=0.48 \pm 0.36$; y dominancia dimensional $U_{1}=0.32 \pm 0.15, U_{2}=0.20 \pm 0.13, U_{3}=0.24 \pm 0.14$ y $U_{4}=0.22 \pm$ 0.12 . Se concluye que los índices de agregación y segregación presentan una distribución aleatoria para los sitios 1 y 4, y una distribución de agrupamiento para los sitios 2 y 3 . Según la uniformidad de ángulos la distribución es regular en todos los sitios. Del análisis de mezcla de especies se infiere que los sitios 1 y 4 muestran una competencia interespecífica, mientras que en los sitios 2 y 3 hay una competencia intraespecífica. En diferenciación diamétrica y dominancia dimensional la estructura de las cuatro áreas fue homogénea. El sitio 4 reúne las características idóneas para ser replicadas en áreas que se encuentran en proceso de restauración.

Palabras clave: Matorral espinoso tamaulipeco, restauración ecológica, índice de distribución espacial, índice de diferenciación, índice de dominancia.

\section{SUMMARY}

In this study we describe the forest structure using the tree spatial distribution in a Tamaulipan thornscrub (MET) area. We utilized a nearest neighbor approximation and computed the aggregation index $(R)$, uniform angle index $(W)$, segregation index $(S)$, species mingling $(M i)$, diameter differentiation (Ti) and dominance (Ui), in order to provide information that can be used in ecological restoration. Our study was located in an area of MET without vegetation removal and no record of productive activity at least in 28 years. For the forest inventory we stablished four squared plots of $1600 \mathrm{~m}^{2}(40 \times 40 \mathrm{~m})$ in which every tree and shrub $\left(d_{0.10} \geq 5 \mathrm{~cm}\right)$ were located and determined species scientific name; besides, we measured the crown diameter $(m)$, total height $(m)$, distance $(m)$ and azimuth to the center of each plot. The main results were, for aggregation index $R_{1}=0.76, R_{2}=0.43, R_{3}=$ 0.50 and $R_{4}=0.83$; uniform angle index $W_{1}=0.57 \pm 0.20, W i_{2}=0.61 \pm 0.24$, $W I_{3}=0.59 \pm 0.23$ and $W_{4}=0.52 \pm 0.20$; segregation index $S_{1}=0.24, S_{2}=$ $0.68, S_{3}=0.66$ and $S_{4}=0.04$; species mingling $M i_{1}=0.73 \pm 0.24, M i_{2}=0.47$ $\pm 0.35, \mathrm{Mi}_{3}=0.52 \pm 0.34$ and $\mathrm{Mi}_{4}=0.84 \pm 0$; diameter differentiation $\mathrm{Ti}_{1,34}$ $=0.49 \pm 0.35$ and $\mathrm{Ti}_{2}=0.48 \pm 0.36$; and diameter dominance $U_{1}=0.32 \pm$ $0.15, U_{2}=0.20 \pm 0.13, U_{3}=0.24 \pm 0.14$ and $U_{4}=0.22 \pm 0.12$. Hence, results based on aggregation and segregation indices show a random distribution in plots 1 and 4, and a clumped distribution in plots 2 and 3 . According to the uniform angle index, we can conclude that this area has a regular distribution in all plots. The analysis of species mingling showed interspecific competition in plots 1 and 4, while in plots 2 and 3 there is an intraspecific competition. Finally, the diametric dominance and differentiation showed homogeneous distribution in all plots. Based on these results, we recommend that the plot 4 can be taken as a reference site because it showed special characteristics that could be replicated on degraded lands.

Index words: Tamaulipan Thornscrub, ecological restoration, spatial distribution index, differentiation index, dominance index.

\section{INTRODUCCIÓN}

La caracterización estructural de una comunidad vegetal es una manera de estimar la condición de los ecosistemas en un momento determinado y su evolución en el tiempo (Gadow et al., 2007, Ni et al., 2014). La estructura de la comunidad es considerada como un indicador de la biodiversidad (Hui y Pommerening, 2014). Para el estudio de la biodiversidad se consideran índices estructurales y variables dendrométricas que incluyen diámetro, altura, área basal, densidad, entre otras, para así lograr una mejor descripción. La caracterización de la estructura de los ecosistemas forestales constituye una condición inicial para la toma de decisiones sobre el manejo sustentable de los recursos naturales (Aguirre et al., 2003; Jiménez et al., 2001).

La estructura de un ecosistema hace referencia a la distribución de las principales características arbóreas en 
el espacio, y de especial importancia es la distribución de las especies por clases de dimensión (Li et al., 2014). Por otro lado, la estructura espacial de una masa es una característica que debe considerarse para una correcta planificación de los recursos naturales. Aunque habitualmente se suele asociar con la distribución de frecuencias del número de árboles por especie y tamaños, en muchos casos el uso exclusivo de estas distribuciones no es suficiente para describir la estructura espacial de una masa (Li et al., 2014; Wehenkel et al., 2014).

El análisis de la distribución espacial, composición y estructura de comunidades vegetales se ha incrementado entre científicos y gestores de los recursos naturales por ser el punto de partida para la correcta planeación de los programas de manejo, conservación y restauración ecológica (Gadow et al., 2012; Ni et al., 2014). En México se han desarrollado estudios que evalúan la estructura de comunidades vegetales, y los ecosistemas de clima templado son los más estudiados (Alanís et al., 2011; Alanís 2012; Ávila et al., 2012; González et al., 2007; González et al., 2008), seguidos de las comunidades vegetales del noreste de México, que se describen con y sin manejo forestal (Corral et al., 2005; García et al., 2013; Hernández et al., 2013; Solís et al., 2006; Torres et al., 2006). Sin embargo, los matorrales han sido escasamente evaluados (Alanís et al., 2008; Jiménez et al., 2012) y los estudios existentes, únicamente analizan comunidades vegetales regeneradas después de disturbios.

En el matorral espinoso tamaulipeco (MET) recientemente se ha generado información fitosociológica y dendrométrica de las especies arbóreas y arbustivas que se regeneran después de la presencia de un disturbio, con el objetivo de evaluar comunidades vegetales resultantes desde el punto de vista de su composición (Jiménez et al., 2013; Molina et al., 2013; Molina et al., 2014; Mora et al., 2014). Estas investigaciones son importantes para evaluar cómo se encuentran las áreas regeneradas postperturbación. Debido a que es limitada la información de comunidades vegetales maduras y sin disturbios que sirvan de referencia para establecer programas de restauración ecológica, el objetivo del presente estudio es describir la distribución espacial, composición y estructura de la vegetación arbórea y arbustiva de una comunidad vegetal madura del matorral espinoso tamaulipeco del noreste de México, mediante índices que utilizan relaciones de vecindad y describen patrones espaciales de distribución, con la finalidad de establecer los parámetros para definir una condición idónea que sirvan como base para restaurar zona similares.

\section{MATERIALES Y MÉTODOS}

\section{Área de estudio}

La presente investigación se desarrolló en la reserva ecológica del Matorral-Escuela de la Facultad de Ciencias Forestales de la Universidad Autónoma de Nuevo León (Figura 1) en el municipio de Linares, Nuevo León (Noreste de México), ubicada en las coordenadas $24^{\circ} 46^{\prime}$ y $56.7^{\prime \prime} \mathrm{N}$ y $99^{\circ} 37^{\prime} 34.3^{\prime \prime} \mathrm{O}$ a una altitud de $366 \mathrm{~m}$. La precipitación media anual va de 350 a $600 \mathrm{~mm}$; la temperatura media anual es de $22.4^{\circ} \mathrm{C}$, con temperaturas en invierno inferiores a 0 ${ }^{\circ} \mathrm{C}$ y en verano superiores a $40{ }^{\circ} \mathrm{C}$.

\section{Análisis de la información}

Se ubicó un área del matorral espinoso tamaulipeco (MET) sin registro histórico de alguna actividad productiva que implicara la remoción total de la vegetación (Figura 2). El área seleccionada se encuentra delimitada por un cerco perimetral desde hace 30 años (1984-2014), donde se tiene la certeza que no se han desarrollado actividades que hayan causado algún tipo de perturbación. En el verano del año 2014 se establecieron cuatro sitios de muestreo con iguales condiciones de vegetación entre ellas. Las dimensiones de los sitios de muestreo fueron de $40 \times 40$ m (1600 $\left.m^{2}\right)$.

En cada sitio de muestreo se realizó un censo de todas las especies arbóreas y arbustivas de diámetro basal $\left(d_{0.10}\right.$ $\geq 5 \mathrm{~cm}$ ), y se registraron las siguientes variables dendrométricas: diámetro basal $\left(d_{0.10}\right)$, que es una medida estándar empleada para especies arbóreas y arbustivas del MET (Alanís et al., 2008), altura total (m), y ubicación mediante la distancia $(\mathrm{m})$ y azimut $\left(^{\circ}\right)$ de cada árbol y arbusto con respecto al centro del sitio de muestreo.

Para describir la estructura se utilizaron índices de relaciones de vecindad y descripción del patrón de puntos en el espacio (Gadow et al., 2007), que se basan en información de los árboles más cercanos. Se usó el método de muestreo estructural de los cinco árboles, desarrollado para evaluar los atributos estructurales (dimensiones, especies y la regularidad de sus posiciones) de los árboles que forman una masa forestal (Aguirre et al., 2003; Gadow et al., 2001).

Los gráficos, análisis y la conformación de los grupos estructurales se hicieron mediante el paquete (CR v2.15, con apoyo en los "Scripts" (comandos) proporcionados por el Woodland Structure Analysis and Modelling Lab. (Pommerening, 2013), se llevaron a cabo todos los análisis de vecindad; y con el soporte de las bibliotecas SPATSTAT (Baddeley y Turner, 2005) se realizó la proyección gráfica 


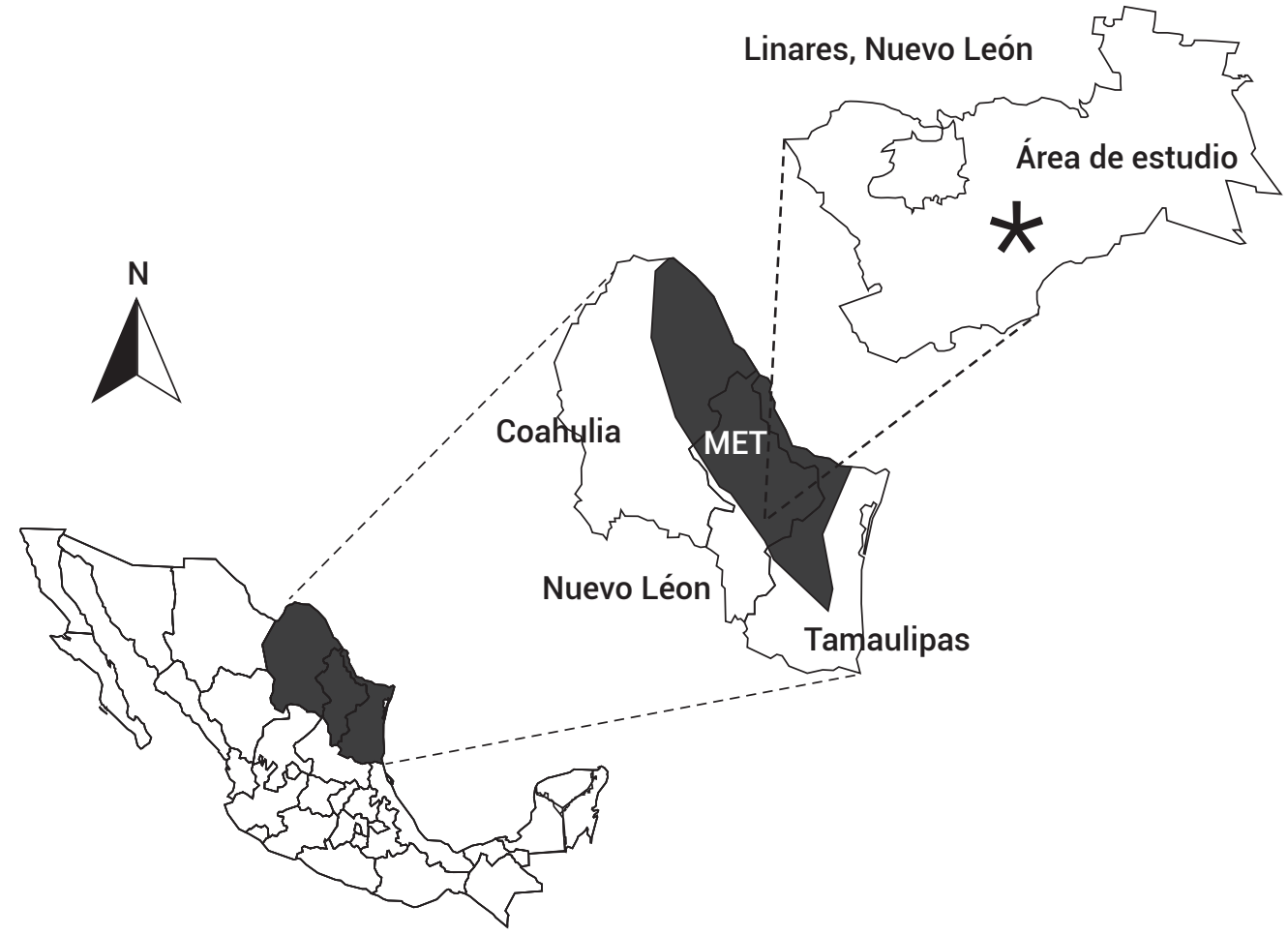

Figura 1. Localización del área de estudio.

del arbolado, y con LATTICE (Sarkar, 2013) se generaron gráficos de distribuciones de los distintos índices que se describen a continuación.

Índice de agregación $R$. Es una medida de agrupamiento de un patrón de puntos. Describe la relación de la distancia media observada ( $r$ observada) del vecino más cercano con respecto a la esperada ( $r$ esperada) en una distribución de puntos tipo Poisson, o aleatoria (Ec. 1) (Clark y Evans, 1954):

$$
R=\frac{\bar{r} \text { observada }}{\bar{r} \text { esperada }}
$$

Teóricamente, $R$ toma valores entre 0 (mayor grado de agrupamiento) y 2.1491 (patrón regular de forma hexagonal), que indica cuan regular, aleatoria o agrupada es la distribución espacial del arbolado en un área determinada. Los valores de agregación menores a 1, cercanos a 1 y mayores a 1 indican agrupamiento, aleatoriedad y distribución regular, respectivamente (Aguirre, 2004; Pommerening, 2002). El valor de $R$ se determina mediante análisis de vecindad con respecto al vecino más cercano, para lo cual se mide la distancia de cada árbol a su vecino más cercano en un área determinada, y la distancia media de cada árbol se calcula con la Ec. 2:

$$
\bar{r} \text { observada }=\frac{\sum_{i=1}^{n} r_{i}}{n}
$$

En tanto, la r esperada se calcula con la Ec. 3:

$$
\bar{r} \text { esperada }=\frac{1}{\sqrt[2]{N / A}}
$$

donde: $A$ = área de la parcela y $N=$ número de árboles en la parcela.

Índice de uniformidad de ángulos $\left(W_{\mathrm{i}}\right)$. Se basa en la medición de los ángulos entre los vecinos a un árbol de referencia i y su comparación con un ángulo estándar a, de tal manera que al considerar cuatro vecinos al árbol de referencia $W_{i}$ puede tomar valores de 0 hasta 1 , donde un valor cercano a cero representa condiciones de regularidad, valores cercanos a 0.5 muestran tendencia a la aleatoriedad y los próximos a 1 presentan condiciones de agrupamiento. $W_{i}$ se calcula con la Ec. 4 (Gadow et al., 1998):

$$
W_{i}=\frac{1}{n} \sum_{j=1}^{n} V_{i j}
$$

donde: $W_{i}=$ valor del índice para el $j$-ésimo árbol referencia, $n=$ es el número de árboles vecinos considerados, $V_{i j}$ = variable 1 cuando el $j$-ésimo ángulo $\alpha$ entre dos árboles vecinos próximos es menor o igual al ángulo estándar $\alpha_{i}$ en caso contrario toma un valor de 0.

Índice de segregación de Pielou (S). Describe la combinación o entremezcla de dos especies de árboles. Al igual que el índice $R$, está basado en las relaciones de vecindad 
(Pielou, 1977)

El índice $S$ de segregación es definido por:

$$
S=7-\frac{N *(b+c)}{m * S+n * r} \quad(\text { EC. } 5)
$$

donde: $\mathrm{N}$ = es el número total de pares de árboles (árbol referencia y su vecino más cercano), $n$ y $m$ son el número de especies $A$ y $B$ respectivamente, $r$ y s son el número de veces que los árboles de las especies A y B se encuentran como los vecinos más cercanos al árbol referencia. Si el vecino más cercano es de la misma especie que el árbol de referencia entonces $S=1$. En caso contrario, si todos los vecinos son de especie diferente a los árboles de referencia, entonces $S=-1$. Los valores cercanos a $S=0$ se puede interpretar como una distribución completamente aleatoria de las especies (Aguirre, 2004; Pommerening, 2002).

La mezcla de especies $\left(M_{i}\right)$. Es una medida de la segregación espacial de los individuos de diferentes especies, donde se define el valor del índice del árbol de referencia $i$ como la proporción de vecinos que pertenecen a especies diferentes a dicho árbol de referencia (Ec. 6) (Füldner, 1995; Gadow y Füldner, 1993;):

$$
M_{j}=\frac{1}{n} \sum_{j=1}^{n} V_{j} \quad \text { (Ec. 6) }
$$

donde: $0 \leq M_{i} \leq 1$ y $V_{i}$ es igual a 0 si el árbol $j$ es de la misma especie que el árbol de referencia $i$, y 1 en caso contrario. De este modo, si se analizan los $n$ vecinos más próximos el grado de mezcla puede tomar $n+1$ valores discretos. Los valores de $M_{j}$ varían de 0 a 1 , de modo que cuanto más próximo a 1 mayor es la mezcla de especies, y cuanto más próximo a 0 más se agrupan los individuos de las especies (Del Río et al., 2003).

Índice de diferenciación dimensional $\left(T_{i}\right)$. Este índice se puede aplicar a cualquier variable que represente el tamaño del árbol, en este caso a diámetros. Toma en cuenta los $n$ árboles más cercanos al árbol de referencia $i$ y se define con la Ec. 7 (Gadow et al., 2007):

$$
T_{i}=1-\frac{1}{n} \sum_{j=1}^{n} \frac{\min (\text { Dimi, Dimj) }}{\max (\text { Dimi, Dimj) }}(\text { Ec. 7) }
$$

donde: $T_{i}=$ Grado de diferenciación en diámetros; $j=1 ; n$ árboles vecinos; $i=$ árbol de referencia; $\operatorname{Dim}_{i}=$ diámetro del árbol $i ; \operatorname{Dim}_{j}=$ diámetro del árbol $j$.

El valor del $T$, se incrementa al aumentar la diferencia media del tamaño de los árboles vecinos $(0 \leq T i \leq 1)$. Entre más bajo sea el valor de $T_{i}$ las diferencias son menores, y un valor de 0 indica que todos los árboles son del mismo tamaño (Gadow et al., 2007). El grado de diferenciación en
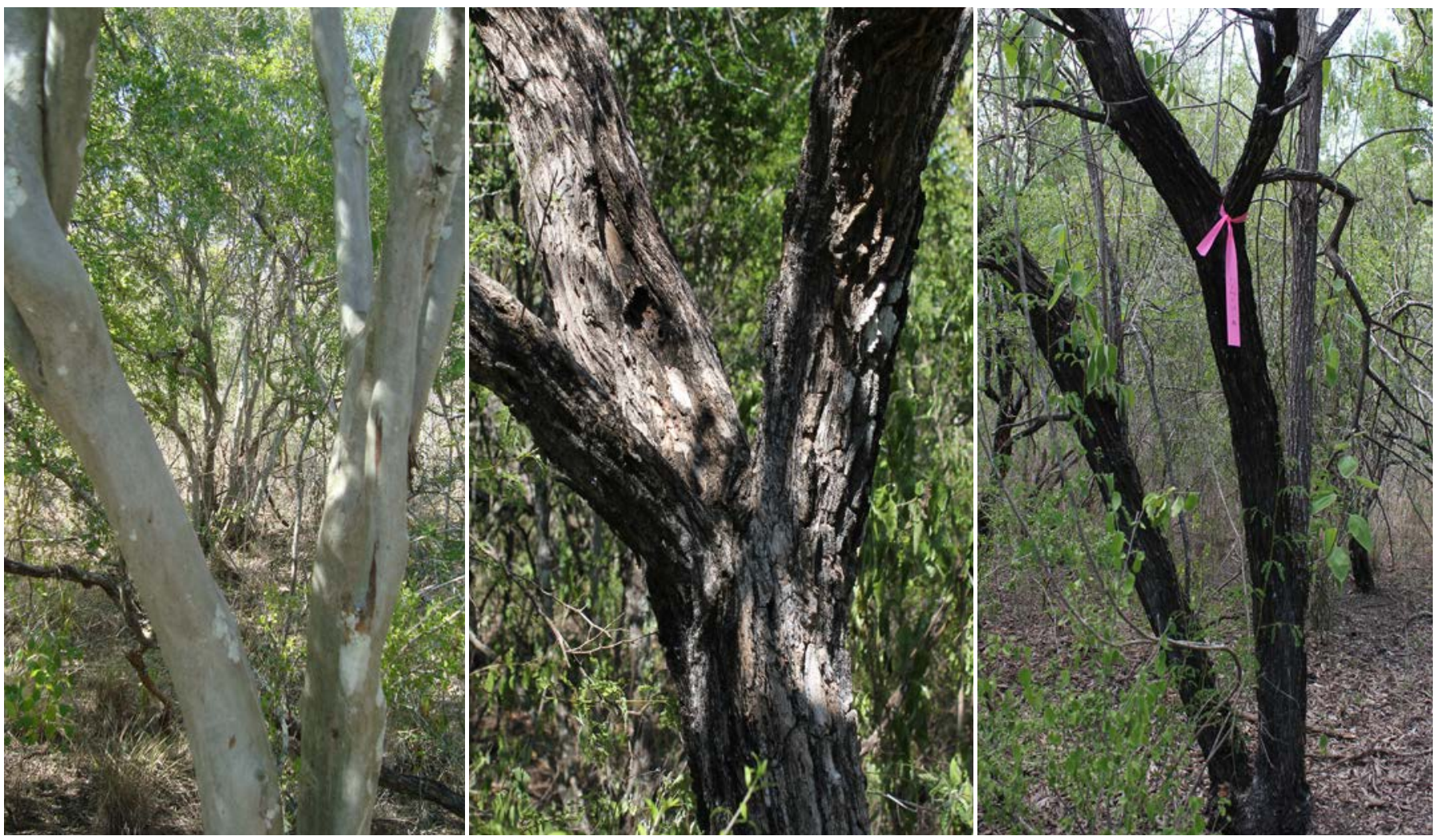

Figura 2. Fotografías ilustrativas del matorral espinoso tamaulipeco. 
diámetros se clasificó en cinco grupos, de 0.0 a 0.2 débil; de 0.2 a 0.4 moderada; de 0.4 a 0.6 clara; de 0.6 a 0.8 fuerte; y de 0.8 a 1.0 muy fuerte (Aguirre et al., 1998; Jiménez et al., 1998).

Índice de dominancia dimensional $\left(U_{j}\right)$. también conocido como medida del entorno, describe la dominancia relativa de una especie en su entorno directo. Se define como la proporción de los $n$ vecinos más próximos al árbol de referencia que son de menor tamaño que él (Ec. 8) (Gadow et al., 2007):

$$
U_{i}=\frac{1}{n} \sum_{j=1}^{n} V_{j}
$$

donde: $0 \leq U i \leq 1$ y $v_{j}$ igual a 1 si el árbol $j$ es menor que el árbol de referencia $i$ y 0 en caso contrario. Con cuatro vecinos $U_{i}$ puede tener cinco valores, que corresponden a las clases sociales propuestas por Kraft (1884): $U_{i}=0.0$, si los cuatro vecinos son más grandes que el árbol referencia $i$ (suprimido); $U i=0.25$, si tres de los vecinos son más grandes (intermedio); $\mathrm{Ui}=0.50$, si dos de los vecinos son más grandes (codominante); $\mathrm{Ui}=0.75$, si uno de los cuatro vecinos es más grande (dominante); y $\mathrm{Ui}=1$, si ninguno de los cuatro vecinos es más grande que el árbol de referencia i (muy dominante).

\section{RESULTADOS Y DISCUSIÓN}

El índice de agregación $(R)$ muestra una tendencia a la aleatoriedad. Los sitios 4 y 1 muestran valores de 0.83 y 0.76 respectivamente, que indican una distribución aleatoria. Los sitios 2 y 3 muestran valores más cercanos al agrupamiento con 0.43 y 0.50 respectivamente (Figura 3 ).

De acuerdo con los resultados el índice de Uniformidad de ángulos $(W)$, la distribución de los individuos presenta una tendencia a la aleatoriedad. Los valores de este índice fueron: $W_{i}=0.57 \pm 0.20, W_{i}=0.61 \pm 0.24, W_{i}=0.59 \pm 0.23 \mathrm{y}$ $W_{i}=0.52 \pm 0.20$ para los sitios 1, 2, 3 y 4 respectivamente, con una media de $0.57 \pm 0.22$. Según Hui y Gadow (2002), valores menores a 0.475 sugieren una distribución regular y aquellos mayores a 0.517 una distribución irregular con tendencia a la formación de grupos. Acorde con ello, los valores absolutos y promedio indican una distribución irregular con tendencia a la agrupación. Tal condición puede ser observada en la distribución de frecuencias por grupo estructural en la que el mayor porcentaje de grupos para cada sitio se ubica en la categoría Wi = 0.50 (Figura 4).

Los resultados del análisis de segregación ( $S$ ) fueron: $S_{i 1}=0.73 \pm 0.24, S_{i 2}=0.47 \pm 0.35, S_{i 3}=0.52 \pm 0.34$ y $S_{i 4}$ $=0.84 \pm 0.21$, con un valor promedio total de $S_{i}=0.64 \pm$ 0.29 . Se puede dilucidar que los sitios 1 y 4 muestran una competencia interespecífica (0.24 y 0.04 , respec- tivamente); es decir, se distribuyen aleatoriamente con tres especies diferentes a la suya; mientras que los sitios 2 y 3 presentan una competencia intraespecífica (0.68 y 0.66 , respectivamente), con una ligera tendencia a una agrupación de solo un individuo o dos de la especie diferente a la suya. Estos resultados coinciden con los de Jiménez et al. (2009) quienes evaluaron un área del MET con distinto historial de uso, pero que también presenta una competencia interespecífica debido a que presentan vecindad con especies diferentes.

Los sitios 1 y 4 tienen mayor frecuencia en la categoría $M_{1}$, lo que indica que ninguno de los cuatro árboles vecinos es de la misma especie al árbol centro (Figura 5); es decir, se puede inferir que estos sitios muestran especies con una alta competencia interespecífica, ya que presentan vecindad con especies diferentes a la suya. Los sitios 2 y 3 muestran un comportamiento distinto al de 1 y 4 , donde existe mayor equidad de frecuencia en los diferentes valores de mezcla. Los resultados para los sitios 1, 3 y $4(0.57,0.59$ y 0.52 respectivamente) son menores a los encontrados por Jiménez et al. (2009), que fueron de 0.62 , 0.63 y 0.64 en diferentes condiciones; en cambio, el sitio 2 fue muy similar (0.61) a sitios con disturbios por ganadería extensiva (0.62).

La distribución de valores del índice de diferenciación en diámetro (Figura 6) muestra que los valores mayores se encuentran en una diferenciación dimensional escasa, que decrecen conforme se incrementa el valor de $T D_{i}$; esto indica que la mayoría de los individuos presenta uniformidad de dimensión. En todos los sitios el valor promedio se encuentra en la categoría de diferenciación clara (0.4 a 0.6). Estos resultados son similares a los reportados por Jiménez et al. (2009), quienes evaluaron comunidades vegetales regeneradas del MET y también registraron uniformidad en la dimensión diamétrica.

Los valores de similitud en la frecuencia de la dominancia diamétrica en los sitios y las categorías del índice de dominancia (Figura 7), indican una gran similitud en los cuatro sitios $\left(U_{i 1}=0.32 \pm 0.15, U_{i 2}=0.20 \pm 0.13, U_{i 3}=0.24 \pm\right.$ 0.14 y $U_{i 4}=0.22 \pm 0.12$, con un promedio de $\left.U_{i}=0.24 \pm 0.13\right)$, ya que según Kraft (1884) los valores de $U_{i}$ cercanos a 0.25 corresponden a la clasificación intermedio en la que dos de los vecinos son iguales que él.

Otros estudios de este tipo efectuados en otros países son el de Noy-Meir et al. (2012) hecho en un bosque espinal en Córdoba, Argentina, y el de Urbieta (2009) quien evaluó la estructura, dinámica y regeneración de los bosques mixtos de Alcornoque, en la Península Ibérica. En los pocos estudios de este tipo efectuados en México, están los de Alanís et al. (2011), Ávila et al. (2012), Ávila et al. (2014) 
Sitio 1

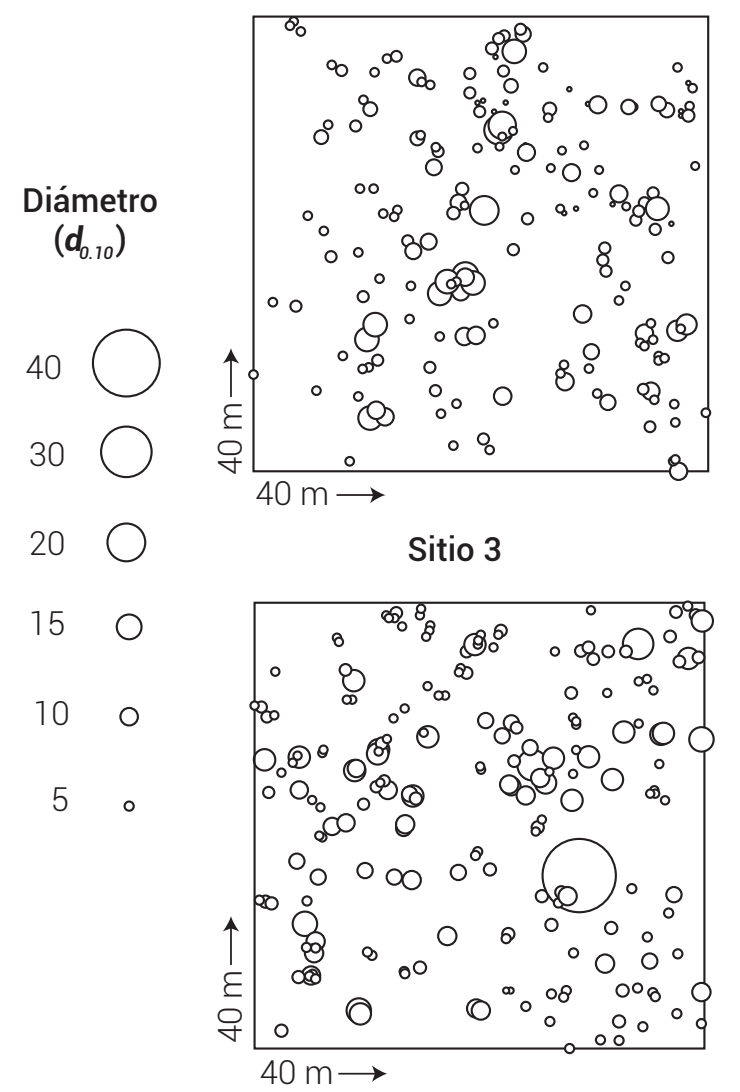

Sitio 2

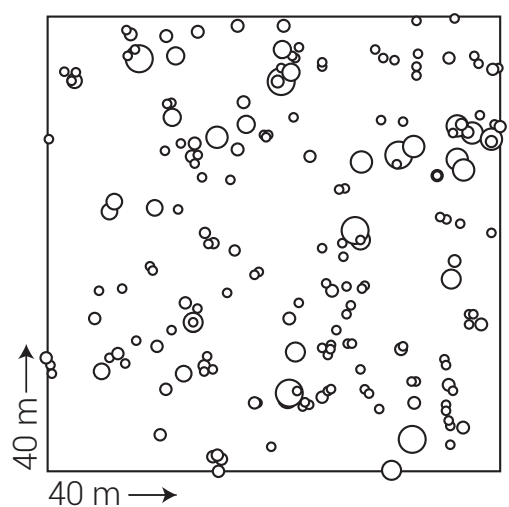

Sitio 4

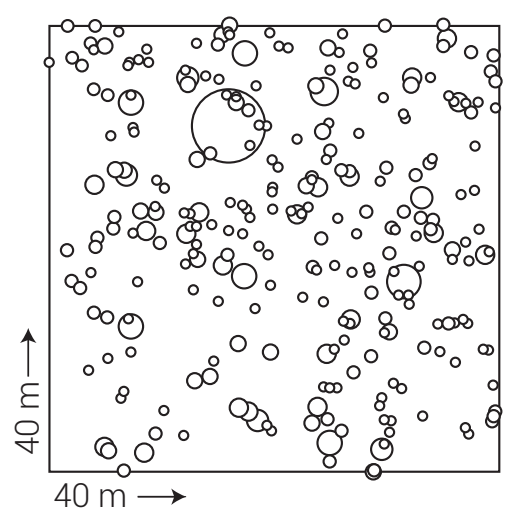

Figura 3. Distribución espacial en cuatro áreas del matorral espinoso tamaulipeco. Los puntos indican la posición relativa de los individuos dentro del área de muestreo.

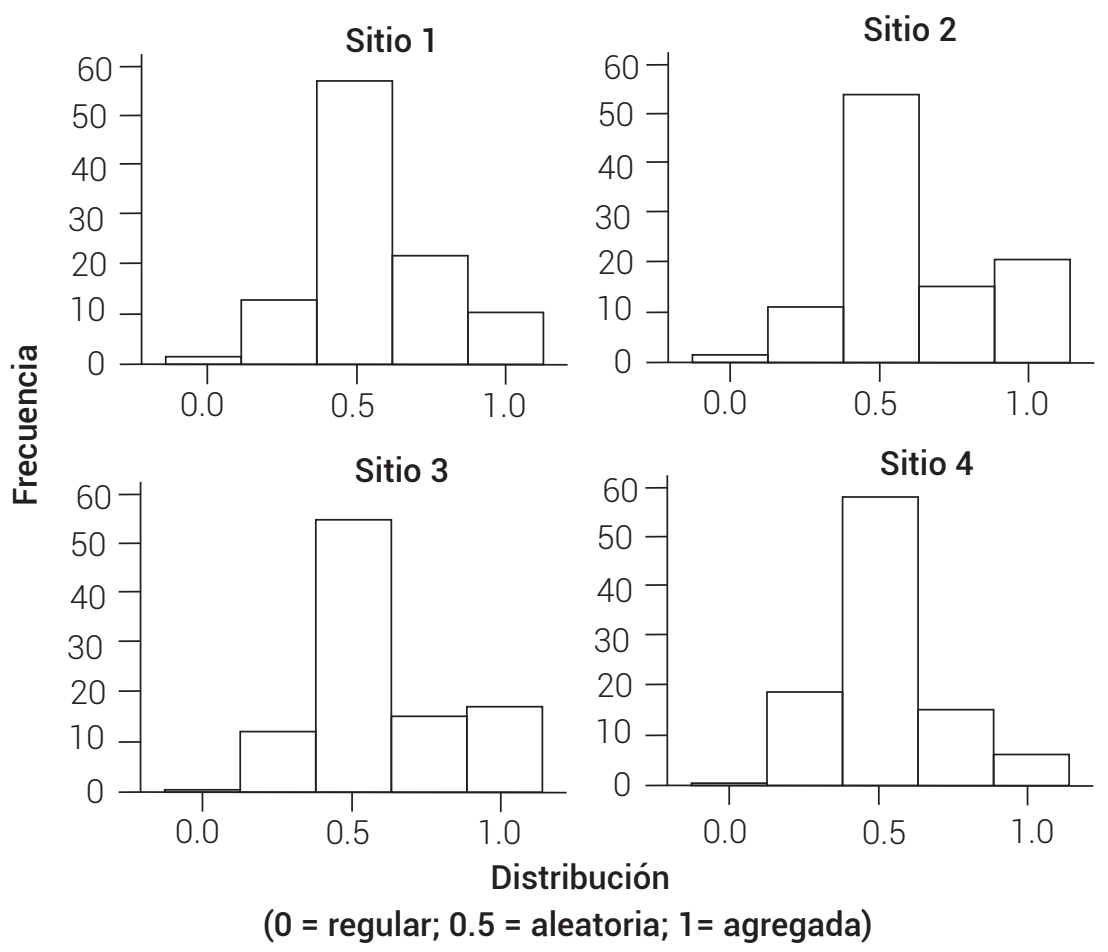

Figura 4. Distribución de frecuencias del índice de uniformidad de ángulos de Gadow para cuatro sitios del MET. 


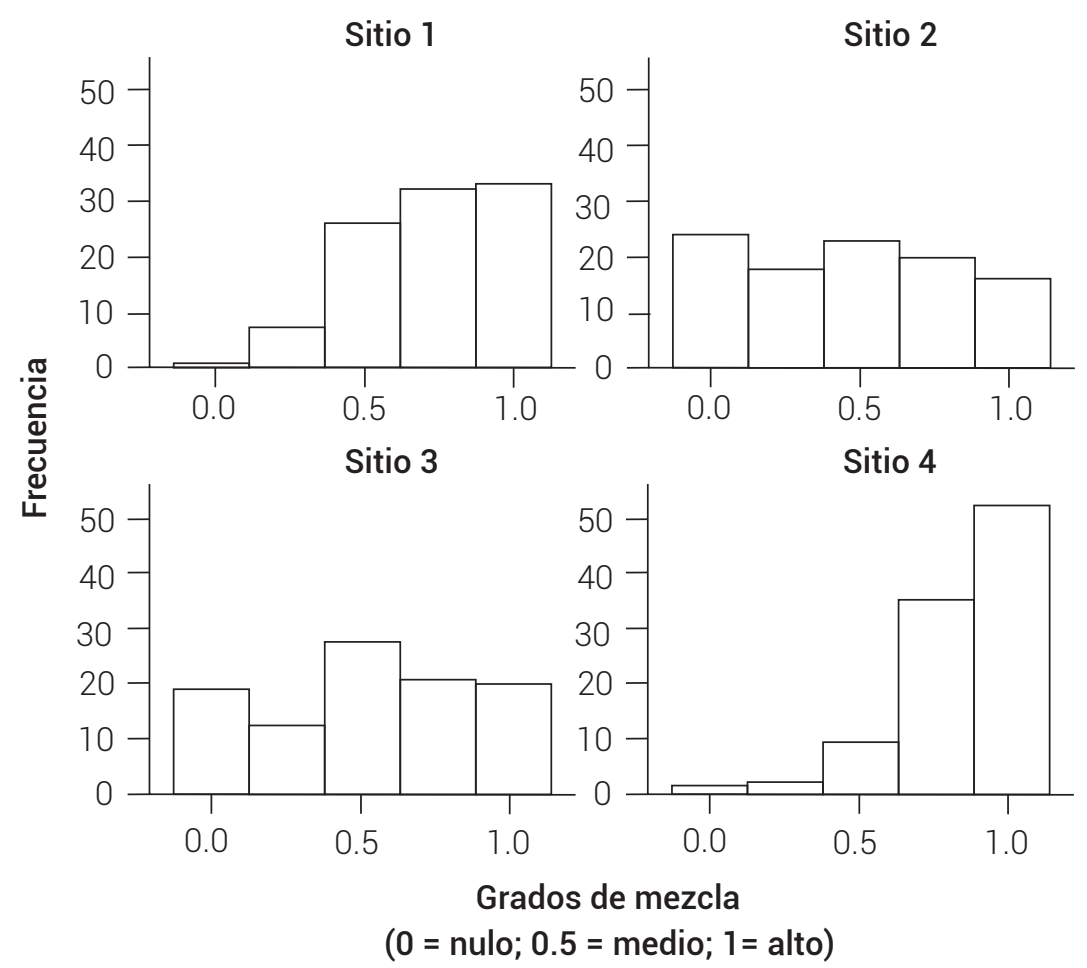

Figura 5. Distribución de la frecuencia porcentual de los valores del índice de mezcla de especies de Gadow en los sitios evaluados.

y Jiménez et al. (2009), todos ellos hechos en ecosistemas templados en el noreste de México, por lo que resulta complicado hacer comparaciones con el MET por tratase de ecosistemas diferentes al aquí estudiado.

Con base en los índices aquí estudiados ( $R=0.83, \mathrm{~W}=$ $0.52, \mathrm{~S}=0.84, \mathrm{M}=0.52, \mathrm{TD}=0.49 \mathrm{y} U=0.22)$, el sitio 4 es el que presenta las mejores condiciones, por lo que este sitio se puede considerar como modelo a seguir para futuros trabajos de restauración en este tipo de vegetación. Estos índices también aportan información relevante acerca de la distribución, la estructura dimensional y las interrelaciones entre especies presentes en un lugar, que los convierte en mejores criterios que los métodos tradicionales basados en el comportamiento individual pero sin considerar el entorno de desarrollo de las especies. Además, la información requerida para aplicar este tipo de índices es sencilla de tomar en campo y con un bajo costo.

\section{CONCLUSIONES}

Los índices utilizados fueron adecuados para la caracterización de este ecosistema, y permitieron hacer las siguientes conclusiones: 1) Los índices de agregación y segregación presentan una distribución aleatoria para los sitios 1 y 4; y una distribución de agrupamiento para los sitios 2 y 3; pero en la uniformidad de ángulos presentan una distribución regular en la que todos los sitios tienden a la agrupación; 2) De acuerdo con el análisis de mezcla de especies, los sitios 1 y 4 muestran competencia interespecífica, mientras que el sitios 2 y 3 presentan competencia intraespecífica; 3)La estructura de las cuatro áreas estudiadas resultaron ser altamente homogéneas en la diferenciación diamétrica y con dominancia dimensional.

Con este estudio se pudo descubrir que el sitio 4 tienen las características idóneas para ser replicado en áreas que se encuentran en proceso de restauración ecológica.

\section{AGRADECIMIENTOS}

A la Facultad de Ciencias Forestales de la Universidad Autónoma de Nuevo León, por las facilidades otorgadas para el establecimiento y desarrollo de la investigación. Al equipo de trabajo de la Facultad, en especial a los compañeros José Ángel Sigala, Román Ramírez y Yazmín Hailen Ugalde, por su apoyo incondicional en el levantamiento de datos en campo. El proyecto fue financiado parcialmente por el proyecto PROMEP/103.5/12/3585.

\section{BIBLIOGRAFIA}

Aguirre 0. A. (2004) Índice para la caracterización del estrato arbóreo de ecosistemas forestales. Revista Ciencia Forestal Mexicana 27:5-27.

Aguirre O. A., H. Kramer and J. Jiménez (1998) Strukturuntersuchungen in Einem Kiefern-Durchforstungsversuch Nordmexikos. Allgemeine Forst u. Jagdzeitung 169:213-220. 
Sitio 1

Sitio 2
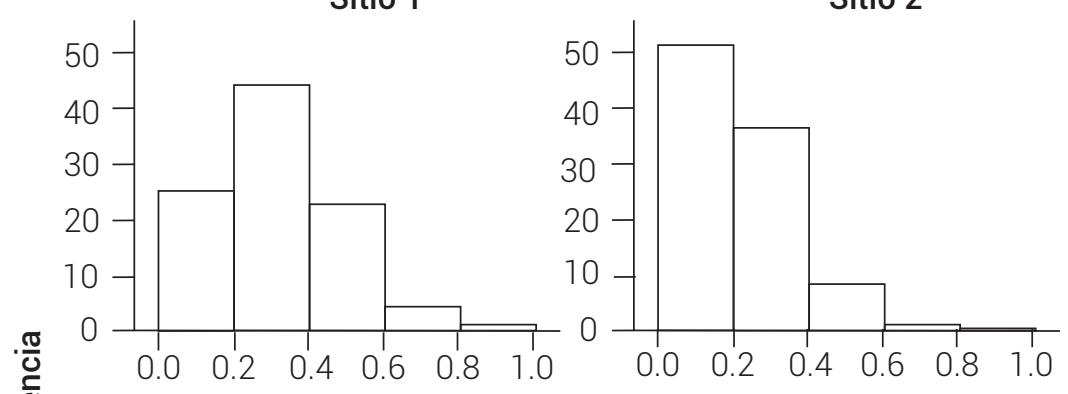

Sitio 3

Sitio 4
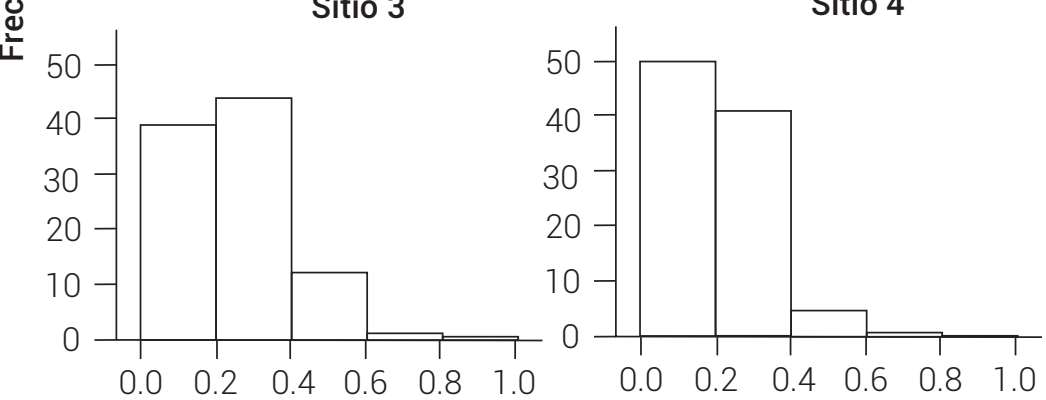

Grados de diferenciación

(0.0-0.2 = débil; $0.2-0.4=$ moderada; $0.4-0.6=$ clara

$0.6-0.8$ = fuerte; $0.8-1.0=$ muy fuerte)

Figura 6. Grado de diferenciación diamétrica (Ti) por sitio en el área de estudio.

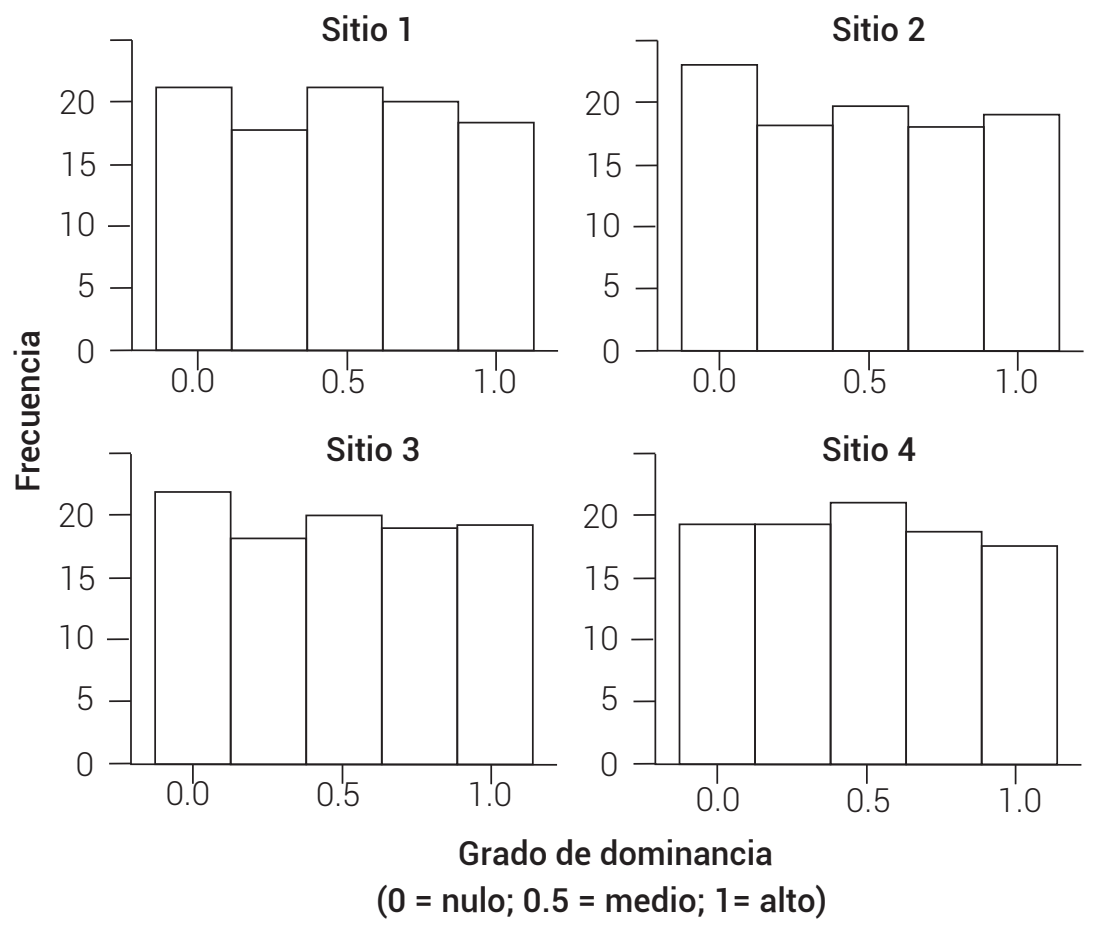

Figura 7. Distribución de la frecuencia porcentual de los valores del grado de dominancia dimensional en diámetro Ui de los sitios evaluados. 
Aguirre O. A., G. Hui, K. Gadow and J. Jiménez (2003) An analysis of spatial forest structure using neighbourhood - based variables. Forest Ecology and Management 183:137-145.

Alanís E. (2012) Regeneración natural y restauración ecológica postincendio de un bosque mixto en el Parque Ecológico Chipinque, México. Ecosistemas 21:206-210.

Alanís E., J. Jiménez, O. A. Aguirre, E. J. Treviño, E. Jurado y M. A. González (2008) Efecto del uso del suelo en la fitodiversidad del matorral espinoso tamaulipeco. Ciencia UANL 11:56-62

Alanís E., J. Jiménez, A. Valdecantos, M. Pando, O. A. Aguirre y E. J. Treviño (2011) Caracterización de regeneración leñosa post-incendio de un ecosistema templado del Parque Ecológico Chipinque, México. Revista Chapingo serie Ciencias Forestales y del Ambiente 17:31-39.

Ávila D. Y., M. A. González, J. Jiménez, O. A. Aguirre, E. J. Treviño y B. Vargas (2012) Evaluación de la estructura espacial post-incendio de rodales de Pinus hartwegii utilizando parámetros de vecindad en la Sierra Madre Oriental, México. Tropical and Subtropical Agroecosystems 15:377-387.

Ávila D. Y., M. A. González, J. Jiménez, O. A. Aguirre, E. J. Treviño, B. Vargas y E. Alanís (2014) Efecto de la severidad del fuego en las caracterís ticas de la estructura forestal en rodales de coníferas. Revista Chapingo Serie Ciencias Forestales y del Ambiente 20:33-45.

Baddeley A. y R. Turner (2005) SPATSTAT: An R package for analyzing spatial point patterns. Journal of Statistical Software 12:1-42.

Corral J. C., O. A. Aguirre, J. Jiménez y S. Corral (2005) Un análisis del efecto del aprovechamiento forestal sobre la diversidad estructural en el bosque mesófilo de montaña "El Cielo", Tamaulipas, México. Investigación Agraria: Sistemas y Recursos Forestales $14: 217-228$

Clark P. J. y F. C. Evans (1954) Distance to nearest neighbor as a measure of spatial relationships in populations. Ecology 35:445-453.

Del Río M., F. Montes, I. Cañellas y G. Montero (2003) Índices de diversidad estructural en masas forestales. Investigación Agraria: Sistemas y Recursos Forestales 12:159-176.

Domínguez T. G., H. González, R. G. Ramírez, A. E. Estrada, I. Cantú, M. V. Gómez, J. A. Villareal, M. S. Alvarado y J. G. Alanís (2013) Diversidad estructural del matorral espinoso tamaulipeco durante las épocas seca y húmeda. Revista Mexicana de Ciencias Forestales 4:106-122.

Füldner K. (1995) Zur Strukturbeschreibung in Mischbeständen. Forstarchiv 66:149-161

Gadow K. V. und K. Fülnder (1993) Zurbestandesbeschreibung in der forsteinrichtung. Forst und Holz 21:601-606.

Gadow K. V., G. Hui und M. Albert (1998) Das Winkelmass - ein Strukturparameterzur Beschreibung der Individualverteilung in Waldbeständen. Centralblattfür das gesamte Forstwesen 115:1-9.

Gadow K. V., P. Real y J. G. Álvarez (2001) Modelización del crecimiento y la evolución de los bosques. IUFRO World Series 12:242.

Gadow K. V., S. Sánchez y J. G. Álvarez (2007) Estructura y Crecimiento del Bosque. Universidad de Göttingen, Alemania. $141 \mathrm{p}$.

Gadow K. V., C. Y. Zhang, C. Wehenkel, A. Pommerening, J. Corral-Rivas, M. Korol, S. Myklush, G. Ying, A. Kiviste and X. H. Zhao, (2012) Forest structure and diversity. In: Continuous Cover Forestry. Springer Netherlands. pp:29-83

García Y., L. Olivares y J. Ramos (2013) Estructura y composición arbórea de un fragmento de bosque mesófilo de montaña en el estado de Veracruz. Revista Chapingo Serie Ciencia Forestal y del Ambiente 19:91-101.

González M. A., L. Schewendenm, J. Jiménez y L. Himmelsbach (2007) Reconstrucción del historial de incendios y estructura forestal en bosques mixtos de pino Encino en la Sierra Madre Oriental. Madera y Bosques 13:51-63.

González M. A., L. Schwendemann, J. Jiménez and R. Schulz (2008) Forest structure and woody plant species composition along a fire chronosequence in mixed pine-oak forest in the Sierra Madre Oriental, Northeast Mexico. Forest Ecology and Management 256:161-167.

Hernández J., O. A. Aguirre, E. Alanís, J. Jiménez, E. J. Treviño, M. A. González, C. Lujan, J. M. Olivas y L. A. Domínguez (2013) Efecto del manejo forestal en la diversidad y composición arbórea de un bosque templado del Noreste de México. Revista Chapingo Serie Ciencia Forestal y del Ambiente 19:189-200.

Hui G. Y. und K. Gadow (2002) Das Winkelman ß - Herleitung des optima- len Standardwinkels. Allgemeine Forst u. Jagdzeitung 173:171176.

Hui G. Y. und A. Pommerening (2014) Analysing tree species and size diversity patterns in multi-species uneven-aged forests of Northern China. Forest Ecology and Management 316:125-138.

Jiménez J., O.A. Aguirre und H. Kramer (1998) Bestandestrukturanalyse im ungleichaltrigen Kiefern-Wacholder- Eichen-Mischwald Nordostenmexikos. Forstarchiv 69:227-234.

Jiménez J., O.A. Aguirre y H. Kramer (2001) Análisis de la estructura horizontal y vertical en un ecosistema multicohortal de pino-encino en el norte de México. Investigación Agraria: Sistema y Recursos Forestales 10:355-366

Jiménez J., E. Alanís, O.A. Aguirre, M. Pando y M. A. González (2009) Análisis sobre el efecto del uso del suelo en la diversidad estructural del matorral espinoso tamaulipeco. Madera y Bosques 15:5-20.

Jiménez J., E. Alanís, J. L. Ruiz, M. A. González, J. I. Yerena, G. y J. G. Alanís (2012) Diversidad de la regeneración leñosa del matorral espinoso tamaulipeco con historial agrícola en el NE de México. Ciencia UANL 15:66-71

Jiménez J., E. Alanís, M. A. González, O. A. Aguirre y E. J. Treviño (2013) Characterizing woody species regeneration in areas with different land history tenure in the Tamaulipan Thornscrub, Mexico. The Southwestern Naturalist 58:299-304.

Kraft, G. (1884) Beiträgezurlehre von den Durchforstungen, Schlagstellungen und Lichtungshieben. Verlang Keindworth, Hannover $147 p$

Li Y., G. Hui., Z. Zhao, Y. Hu. and S. Ye (2014) Spatial structural characteristics of three hardwood species in Korean pine broad-leaved forest-Validating the bivariate distribution of structural parameters from the point of tree population. Forest Ecology and Management 314:17-25.

Molina V. M., M. Pando, E. Alanís, P. Canizales, H. González y J. Jiménez (2013) Composición y diversidad vegetal de dos sistemas de pastoreo en el matorral espinoso tamaulipeco del Noreste de México. Revista Mexicana de Ciencias Pecuarias 4:361-371.

Molina V. M., L. Rechy, A. Alcalá, D. Marín y E. Alanís (2014) Composición y diversidad vegetal del matorral mediano subinerme del noreste de México. Revista Iberoamericana de Ciencias 1:111-119.

Mora C. A., E. A. Rubio, E. Alanís, J. Jiménez, M. A. González, M. Mata y A. Mora (2014) Composición y diversidad vegetal de un área de matorral desértico micrófilo con historial pecuario en el noreste de México. Polibotánica 38:53-66.

Ni R., Y. Baiketuerhan, C. Zhang, C., X. Zhao and K. V. Gadow (2014) Analys ing structural diversity in two temperate forests in northeastern China. Forest Ecology and Management. 316:139-147.

Noy-Meir I., M. Masco, M. A. Georgis, D. E. Gurvich, D. Perazzolo y G. Ruiz (2012) Estructura y diversidad de dos fragmentos de bosque espinal en Córdoba, un ecosistema amenazado. Boletín de la Sociedad Argentina de Botánica 47:119-133.

Pielou E. C. (1977) Mathematical Ecology. John Wiley and Sons. 385 p.

Pommerening A. (2002) Approaches to quantifying forest structures. Forestry 75:305-324.

Pommerening A. (2013) Woodland Structure Analysis and Modelling Lab. [En línea]: http://www.crancod.org/wiki/index.php?title=Main Page\&redirect $=$ no (Septiembre 2013)

Sarkar D. (2013) Lattice Graphics. Package 'lattice'. [En línea]: http:// cran.rproject.org/web/packages/lattice/lattice.pdf. (Septiembre 2013).

Solís R., O. A. Aguirre, E. J. Treviño, J. Jiménez, E. Jurado y J. Corral (2006) Efecto de dos tratamientos silvícolas en la estructura de ecosistemas forestales en Durango, México. Madera y Bosques 12:49-64.

Torres E., J. A. Sánchez y J. Jiménez (2006) Análisis estructural de un ecosistema forestal de Pinus-Quercus en la sierra madre oriental. Revista Ciencia Forestal en México 31:7-30.

Urbieta I. R. (2009) Estructura, dinámica y regeneración de los bosques mixtos de Alcornoque (Quercus suber L.) y Quejigo Moruno $(Q$. canariensis Willd.) del sur de la Península Ibérica: una aproximación multiescala. Ecosistemas 18:116-121.

Wehenkel C., J. J. Corral-Rivas and K. V. Gadow (2014) Quantifying differences between ecosystems with particular reference to selection forests in Durango/Mexico. Forest Ecology and Management 316:117-124. 